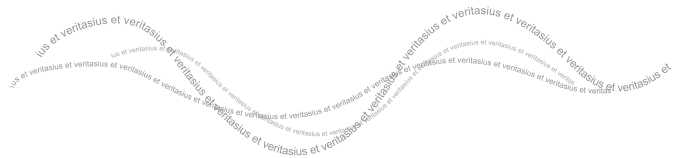

\title{
Diálogos posibles en la investigación de la violencia sexual: Estándares interamericanos y el caso peruano(*)
}

\section{Possible dialogues in the investigation of the sexual violence: Interamerican standards and the Peruvian case}

\author{
Julissa Mantilla Falcón ${ }^{(*)}$ \\ Perú - Pontificia Universidad Católica del Perú
}

\begin{abstract}
Resumen: Los avances en el Sistema Interamericano de Derechos Humanos han permitido ampliar el concepto de violencia sexual y visibilizarlo como una violación a los derechos humanos, de manera que se generen estándares jurídicos para brindar mayor protección a las víctimas. Este artículo plantea el diálogo entre estos estándares interamericanos y el caso peruano en la época del conflicto armado interno, reflejado en el informe final de la Comisión de la Verdad y la Reconciliación, y cómo este ha generado un impacto en la jurisprudencia interamericana de derechos humanos.
\end{abstract}

Palabras clave: Violencia sexual - Derechos Humanos - Comisión de la Verdad y la Reconciliación - Conflicto Armado Peruano - CIDH - Género

\begin{abstract}
The progress of the Interamerican Human Rights System has permitted expand the concept of sexual violence and visualize it as a violation of the human rights, in order to get legal standards to give more protection for victims. This article raises the dialogue between these inter-american standards and the Peruvian case in the period of internal armed conflict, reflected in the final report of the Commission of Truth and Reconciliation, and how this has generated an impact in the inter-american jurisprudence of human rights.
\end{abstract}

Keywords: Sexual violence - Human Rights - Commission of the Truth and Reconciliation - Peruvian Armed Conflict - IACHR - Gender

$\left.{ }^{*}\right) \quad$ Nota del Editor: este artículo fue recibido el 9 de octubre de 2019 y su publicación fue aprobada el 1 de noviembre de 2019.

${ }^{* *}$ Julissa Mantilla Falcón. Lima, Perú. Abogada. LLM en Derecho Internacional de los Derechos Humanos por The London School of Economics and Political Science de la Universidad de Londres. Docente del Departamento de Derecho de la Pontificia Universidad Católica del Perú (PUCP) y de la Academia de Derechos Humanos y Derecho Internacional Humanitario del Washington College of Law de American University (EEUU). Integrante del Grupo de Investigación sobre Protección Internacional de los Derechos de las Personas y los Pueblos (PRIDEP-PUCP). Código ORCID: 0000-0003-2684-3156. La autora agradece a Karen Anaya Cortez y Karen Lopez Retuerto, asistentes de investigación y docencia, por su contribución con insumos para la elaboración de este texto. Miembro extraordinario de lus et Veritas. Correo: jmantil@pucp.edu.pe 


\section{Presentación}

El desarrollo del Sistema Interamericano de Derechos Humanos (SIDH) ha sido de suma importancia tanto para el reconocimiento de los derechos de las personas como para el establecimiento de estándares jurídicos que permitan la investigación de las violaciones de derechos humanos, la determinación de la responsabilidad de los perpetradores y las reparaciones para las víctimas.

Ahora bien, el caso específico de la violencia sexual fue recogido recientemente por el SIDH, lo cual se enmarca en una visión tradicional de las violaciones de derechos humanos que acompañó el desarrollo normativo y jurisprudencial del Derecho Internacional de los Derechos Humanos (DIDH).

Para ello, fue necesario un diálogo e intercambio entre los desarrollos del SIDH y los avances locales, producto del cual nuevos estándares se han ido consolidando tanto para la identificación de la violencia sexual como una violación de derechos humanos como para su investigación efectiva en el cumplimiento del principio de debida diligencia de los Estados. Este artículo, precisamente, plantea este diálogo a partir del caso peruano, el cual recogió los avances de la Comisión Interamericana de Derechos Humanos y produjo herramientas de investigación y construcción de contexto en violencia sexual que posteriormente tuvieron un impacto en la jurisprudencia interamericana en materia de derechos humanos.

\section{La violencia sexual y el trabajo de la Comisión de la Verdad y Reconciliación del Perú (CVR)}

Como se sabe, la CVR fue creada en el 2001 y laboró hasta el 2003, con el mandato específico de investigar los crímenes y graves violaciones de derechos humanos ocurridos entre 1980 y el 2000 , es decir, "asesinatos y secuestros; las desapariciones forzadas; las torturas y otras lesiones graves y las violaciones a los derechos colectivos de las comunidades andinas y nativas del país". Adicionalmente, la CVR debía investigar "otros crímenes y graves violaciones contra los derechos de las personas"(1).

En ese momento, las violaciones de derechos humanos incluidas en su mandato eran las que en ese momento se conocían e identificaban como violaciones graves a nivel nacional e internacional y, por tanto, era lógico que la CVR los priorizara. Sin embargo, no se había planteado la posibilidad de investigar la violencia sexual, ya que solo se había incluido la violación sexual en la base de datos de la CVR, y la idea general en ese momento era que estos hechos no habían sucedido en una magnitud tal que justificara priorizarlos en la investigación. Por ello, la violencia sexual no solo no fue incluida expresamente en el mandato, sino que tampoco fue contemplada en la metodología de trabajo, en los sistemas de información, en la estrategia de comunicaciones, ni en el resto del trabajo de la CVR.

Esta omisión correspondía a la percepción general en ese momento de que los casos de violencia sexual habían ocurrido, pero de manera aislada, ya que los informes de derechos humanos a nivel nacional no los habían contemplado, no existían denuncias ni procesos judiciales sobre estos hechos y los estándares en derechos humanos no contemplaban el tema. Si bien la jurisprudencia de los Tribunales Penales Internacionales de la ex Yugoslavia y de Rwanda ya existían, estos fallos judiciales no eran aún tan conocidos y, en muchos casos, se consideraban ajenos al caso peruano.

Por otro lado, el desarrollo general de la justicia transicional no contemplaba un enfoque de género $y$, en materia de Comisiones de la Verdad, solo las de Guatemala y Sudáfrica habían tenido referencias al caso de las mujeres y la violencia sexual. Por ello, la CVR inicia su trabajo sin un enfoque de género, sin una obligación establecida en el mandato de investigar la violencia sexual y con una base de datos que incluía solo la violación sexual.

Meses después de su creación, y debido al trabajo de las organizaciones de mujeres y las académicas expertas en género, la CVR decide crear una Línea de Género con el encargo de incorporar una perspectiva de género en su trabajo y de visibilizar todas las formas de violencia sexual. En ese momento, los precedentes internacionales indicaban que la violencia sexual se solía presentar en situaciones de conflicto armado y extrema violencia, y que la falta de denuncia de las víctimas se daba por miedo al estigma y la culpa, además de la condena social, lo cual impedía de facto la visibilización e investigación de estos hechos. Ese fue el marco para construir las hipótesis de trabajo y

(1) Decreto Supremo No 065/2001/PCM del 4 de junio del 2001. 
una metodología para el caso peruano que significó, en primer lugar, trabajar con una definición amplia de violencia sexual, es decir, que no se limitara a la violación sexual sino que incluyera el acoso sexual, el aborto forzado, los desnudos forzados, las amenazas de violación, entre otros casos, lo cual permitía tener el contexto general de los hechos, e incluir numerosas formas de agresión sexual que habían sido tradicionalmente ignoradas por las investigaciones de derechos humanos.

Asimismo, la CVR sostuvo que las experiencias de las mujeres habían sido invisibilizadas durante las investigaciones sobre el conflicto peruano y su rol se había concentrado en su papel como familiares de las víctimas, lo cual hacía necesario un reconocimiento específico de sus derechos y las afectaciones a los mismos. En ese sentido, la Línea de Género organizó una estrategia de capacitación para los y las funcionarias de la CVR en materia de género, además de una investigación específica de la violencia sexual, destacando que -si bien cualquier persona puede sufrir estos hechos- las mujeres y niñas habían sido víctimas mayoritarias de este tipo de violencia que, además, es una forma de discriminación ${ }^{(2)}$.

Ahora bien, en un contexto político difícil como el que se vivía en esa época, sin denuncias, informes de derechos humanos ni precedentes judiciales y sin la obligación expresa de investigar la violencia sexual en el mandato, la Línea de Género tuvo que desarrollar un trabajo de investigación que le permitiera argumentar por qué debía investigarse la violencia sexual en el conflicto armado peruano. En este punto, el aporte del Sistema Interamericano de Derechos Humanos (SIDH) fue de especial relevancia como se verá a continuación.

\section{El Informe Raquel Martín de Mejía de la Comisión Interamericana de Derechos Humanos (CIDH)}

Como se dijo líneas arriba, al momento en que la CVR desarrollaba su trabajo no se había producido la jurisprudencia de la Corte Interamericana de Derechos Humanos (La Corte) que actualmente existe y que no solo califica la violencia sexual como una violación de derechos humanos, sino que condena los estereotipos de género, hace un análisis diferenciado de los hechos y brinda lineamientos para la investigación. Sin embargo, en 1996 la CIDH había producido el Informe sobre el caso Raquel Martin de Mejía v. el Perú(3), el cual fue de especial relevancia para el trabajo de la CVR y cuyos aportes resumimos a continuación.

En 1989, varios militares ingresaron a la fuerza a la casa de la señora Martin de Mejía en Oxapampa, en busca de su esposo Fernando Mejía Egocheaga, abogado, periodista y activista político de izquierda, quien fue golpeado y subido a la fuerza a una camioneta militar. Posteriormente, diez militares con los rostros cubiertos volvieron a la casa y uno de ellos solicitó entrar para buscar los documentos de identidad del señor Mejía. Al ingresar, el sujeto acusó a la señora Raquel y a su esposo de ser integrantes del Movimiento Revolucionario Túpac Amaru (MRTA) y luego la violó sexualmente. A los 20 minutos, el sujeto regresó para informarle que su esposo sería llevado a Lima y nuevamente la violó.

El análisis que hace la CIDH del caso de la violación sexual sufrida por la señora Raquel Martín de Mejía tiene una serie de aspectos importantes ${ }^{(4)}$, sobre los que nos ocupamos a continuación.

3.1. Las presunciones que realiza la $\mathrm{CIDH}$ Luego de admitir la petición, la CIDH inicia el análisis de los hechos y realiza una serie de presunciones en el tema, de las cuales destacaremos las que se vinculan con la violación sexual sufrida por la víctima.

En primer lugar, la CIDH decide presumir como verdaderos los hechos relativos a la violación sexual de Raquel Martin de Mejía por integrantes del Ejército peruano ${ }^{(5)}$. Este punto fue una contribución importante para el trabajo de la CVR, ya que, además de lo sostenido por los peticionarios, la $\mathrm{CIDH}$ utilizó en su análisis los informes de diversas organizaciones intergubernamentales y no

(2) Más información sobre este tema puede encontrarse en Mantilla Falcón, Julissa (2006). La Comisión de la Verdad y Reconciliación en el Perú y la perspectiva de género: principales logros y hallazgos. Revista IIDH, 43.

(3) Raquel Martin de Mejía v. Perú, Caso 10.970 Informe No. 5/96, Inter-Am.C.H.R., OEA/Ser.L/V/II.91 Doc. 7 at 168 (1996).

(4) Es importante resaltar que cuando se inició el procedimiento ante la CIDH en 1992, el Estado indicó que se trataba de una reiteración del caso 10.466 en el que se le había condenado por la desaparición de Fernando Mejía y Aladino Melgarejo, el cual había sido publicado en el Informe Anual 1990-1991 de la CIDH. No obstante, la Cidh concluyó que sí tenía competencia para pronunciarse sobre las violaciones a los derechos de la señora Martín de Mejía, ya que la petición referida no los incluía. Raquel Martin de Mejía v. Perú, Caso 10.970 Informe No. 5/96, Inter-Am.C.H.R., OEA/Ser.L/V/II.91 Doc. 7 at 168 (1996).

(5) Raquel Martin de Mejía v. Perú, Caso 10.970 Informe No. 5/96, Inter-Am.C.H.R., OEA/Ser.L/V/II.91 Doc. 7 at 168 (1996). 
gubernamentales, los cuales documentaban "la existencia de numerosas violaciones de mujeres en Perú perpetradas por miembros de las fuerzas de seguridad en zonas de emergencia y donde se hace referencia al caso concreto de Raquel Mejía, describiéndolo como representativo de esta situación". A continuación, enumeramos los informes que la $\mathrm{CIDH}$ revisó y que, como veremos posteriormente, fueron de utilidad para el trabajo de la CVR:

a) Relator Especial contra la Tortura:

- Informe de $1992^{(6)}$ : Señalaba que, en las áreas sujetas a estado de emergencia en el Perú, los efectivos militares recurrían al abuso sexual frecuentemente.

- Informe de 1993: Señalaba que se había recibido "abundante información sobre la práctica de la violación y la agresión sexual de que con frecuencia son víctimas las mujeres en el marco de la campaña de las fuerzas de seguridad contra los grupos insurgentes (...) En las zonas de estado de emergencia (...) la violación parece utilizarse como forma de intimidación o castigo contra grupos de civiles sospechosos de colaborar con los grupos insurgentes (...)"(7). Asimismo, el Informe citaba que "El abuso sexual y la violación parecen (...) ser habituales en las zonas en estado de emergencia”(8).

b) Amnistía Internacional (Al):

- Informe de 1986: Al sostiene que había recibido información sobre abuso sexual contra las mujeres en las zonas de emergencia, acciones que permanecían en impunidad total (Amnesty International, 1991b).

- Informe de 1991: Al denunció "una práctica extendida de violaciones cometidas por militares en las distintas incursiones que estos realizan en las comunidades campesinas" (Amnesty International, 1991a).

c) Human Rights Watch (HRW): En un informe de 1992, HRW sostuvo que la violación sexual contra las mujeres peruanas era una práctica común y que los soldados "usan la violencia sexual como un arma para castigar, intimidar, coercionar, humillar y degradar" y que "para una mujer vivir en una cierta área implica correr el riesgo de ser violada, habitualmente por ser considerada sospechosa de pertenecer a la insurgencia" (Human Rights Watch et al., 1992, pág. 2-3). Asimismo, este reporte documentaba más de cuarenta casos de abusos sexuales entre 1989 y 1992 en el Perú, incluyendo el caso de la señora Raquel Martín de Mejía. d) Revista "Caretas": Artículo del 11 de marzo de 1993 que incluía el caso de la señora Martín de Mejía y hacía referencia a una carta del 2 de marzo de ese año dirigida por 23 senadores del Congreso de los Estados Unidos al entonces presidente Alberto Fujimori, en la cual se expresaba la preocupación por la violación sexual que sufrían las mujeres por parte de las fuerzas armadas y se incluía específicamente el caso de la señora Martín de Mejía (Caretas, 1993).

En segundo término, la CIDH presume la inexistencia de recursos internos efectivos tanto para la señora Raquel como para su esposo. En este punto es importante resaltar que, al momento de denunciar la desaparición de Fernando Mejía, la señora Raquel no denunció los abusos sexuales sufridos "temerosa de que la revelación de las violaciones cometidas contra mi persona pudieran causarme ostracismo y exponerme a mayor peligro o daño físico"(9) y porque consideraba que no existían recursos internos efectivos para denunciar este tipo de delitos, sobre todo en las zonas de emergencia donde las fuerzas del orden ejercían la autoridad total.

Nuevamente, la CIDH hizo una revisión de los informes y documentos de distintas entidades para probar que las mujeres víctimas de violación sexual por integrantes de las fuerzas de seguridad y de la policía no tenían acceso a un remedio interno efectivo que las protegiera y les permitiera acceder a la justicia en el Perú. En este punto, los informes concluían que en la época de los hechos no existían procesos judiciales ni investigaciones efectivas contra los miembros de las fuerzas de seguridad acusados por las víctimas de violación sexual, existiendo un halo de tolerancia sobre estos hechos, reforzado por el hecho de la aplicación de la jurisdicción militar en estos $\operatorname{casos}^{(10)}$. Asimismo, estos

(6) N.U., E/CN.4/1993/26, párr. 355. Citado por Raquel Martin de Mejía v. Perú, Caso 10.970 Informe No. 5/96, Inter-Am.C.H.R., OEA/ Ser.L/V/II.91 Doc. 7 at 168 (1996).

(7) N.U., E/CN.4/1994/31, párr. 431 y 432. Citado por Raquel Martin de Mejía v. Perú, Caso 10.970 Informe No. 5/96, Inter-Am.C.H.R., OEA/Ser.L/V/II.91 Doc. 7 at 168 (1996).

(8) N.U., E/CN.4/1994/31, párr. 429, Citado por Raquel Martin de Mejía v. Perú, Caso 10.970 Informe No. 5/96, Inter-Am.C.H.R., OEA/ Ser.L/V/II.91 Doc. 7 at 168 (1996).

(9) Raquel Martin de Mejía v. Perú, Caso 10.970 Informe No. 5/96, Inter-Am.C.H.R., OEA/Ser.L/V/II.91 Doc. 7 at 168 (1996). 
informes afirmaban que las mujeres víctimas no denunciaban fundamentalmente por temor a la humillación pública y porque consideraban que los responsables nunca serían sancionados.

Adicionalmente, la CIDH hizo mención de a una declaración del entonces presidente Fujimori, quien había señalado que "En los casos de violaciones de mujeres, espero que existan investigaciones. Existe una lamentable tradición de impunidad en Perú" (Brooke, 1993).

\subsection{El análisis de fondo}

El aspecto más resaltante de este Informe es el análisis que hace la CIDH de los hechos, a fin de identificar si los abusos sexuales sufridos por la señora Raquel Martín eran una violación del derecho a la integridad personal (artículo 5) y a la intimidad (artículo 11), en relación con la obligación consagrada en el artículo 1.1 de la Convención Americana de Derechos Humanos $(\mathrm{CADH})^{(11)}$ que consagra que los Estados Partes deben respetar los derechos y libertades reconocidos en el Pacto y garantizar su libre y pleno ejercicio a toda persona que esté sujeta a su jurisdicción, sin discriminación alguna ${ }^{(12)}$.

En este punto, la CIDH inicia su análisis haciendo referencia al Derecho Internacional Humanitario (DIH) y al Derecho Penal Internacional (DPI) para resaltar la existencia de una prohibición de los abusos sexuales en épocas de conflictos armados, haciendo mención de los Convenios de Ginebra de 1949, los Protocolos Adicionales de 1977 y al Tribunal Internacional para la ex Yugoslavia de 1993. Posteriormente, la CIDH establece tres elementos que deben conjugarse para que exista tortura, esto es: que se trate de un acto a través del cual se inflijan a una persona penas y sufrimientos físicos y mentales; que el acto sea cometido con un fin y que el agente sea un funcionario público o una persona privada a instigación del primero. En este marco, la CIDH ubica el caso de la señora Raquel Martin de Mejía.

Es interesante el análisis que hace la $\mathrm{CIDH}$ ya que, utilizando doctrina y normativa internacional, concluye, en primer lugar, que la violación sexual ocurrida ocasionó en la víctima sufrimientos físicos y mentales que incluían el miedo a la humillación pública. En segundo lugar, la CIDH verificó el elemento de la intención en el castigo y la intimidación que los agresores quisieron impartirle a la señora Raquel Martín, ya que, al agredirla, el perpetrador le indicó que ella también era requerida por ser subversiva y se vinculó su agresión con las actividades de su esposo. Como tercer requisito, la $\mathrm{CIDH}$ verificó que el acto fue realizado por un integrante de las fuerzas estatales de seguridad. Con este análisis, la $\mathrm{CIDH}$ concluyó que el Estado peruano era responsable de la violación de la prohibición de la tortura contenida en el artículo 5 de la $\mathrm{CADH}^{(13)}$, que sostiene:

"Artículo 5

1. Toda persona tiene derecho a que se respete su integridad física, psíquica y moral.

2. Nadie debe ser sometido a torturas ni a penas o tratos crueles, inhumanos o degradantes. Toda persona privada de libertad será tratada con el respeto debido a la dignidad inherente al ser humano."

Adicionalmente, la $\mathrm{CIDH}$ estableció que la violación sexual sufrida por Raquel Martín también violentaba el derecho a su intimidad contenida en el artículo 11 de la $\mathrm{CADH}^{(14)}$, utilizando en su argumentación estándares establecidos tanto por el Relator Especial contra la Tortura y la Corte Europea de Derechos Humanos. En este último caso, la $\mathrm{CIDH}$ hizo referencia al caso $X$ and $Y$ v. The Netherlands de $1980^{(15)}$, en el que la Corte Europea señaló que "el concepto de vida privada alcanza a la integridad física y moral de una persona, y en consecuencia incluye su vida sexual".

En resumen, la $\mathrm{CIDH}$ declaró que el Estado peruano era responsable de "la violación del derecho a la integridad personal, del derecho a la protección de la honra y la dignidad, del derecho a un recurso efectivo y al debido proceso legal que garantizan, respectivamente, los artículos 5, 11, 25 y 8 de la Convención Americana, así como de la obligación general de respetar y garantizar el ejercicio de esos derechos de acuerdo con el artículo 1.1 de la misma Convención".

(10) Ibidem.

(11) Si bien la Cidh también analiza si hubo violaciones al derecho al debido proceso (artículo 8) y a la protección judicial (artículo 25), para este texto solo nos centraremos en los artículos mencionados en el texto.

(12) Artículo 1.1, Convención Americana de Derechos Humanos, 1979, https://www.oas.org/dil/esp/tratados_b-32_convencion_americana_ sobre_derechos_humanos.htm

(13) Convención Americana de Derechos Humanos, 1979, https://www.oas.org/dil/esp/tratados_b-32_convencion_americana_sobre_ derechos humanos.htm

(14) Convención Americana de Derechos Humanos, 1979, https://www.oas.org/dil/esp/tratados_b-32_convencion_americana_sobre_ derechos humanos.htm

(15) Caso $X$ and $Y$ v. The Netherlands, Application 8978/80, Serie A, No. 167. Citado por Raquel Martin de Mejía v. Perú, Caso 10.970 Informe No. 5/96, Inter-Am.C.H.R., OEA/Ser.L/V/II.91 Doc. 7 at 168 (1996). 
Por tanto, la CIDH recomendó al Estado que realizara una investigación rápida e imparcial de los abusos sexuales sufridos por Raquel Martín de Mejía y que se le diera una justa indemnización, entre otras medidas.

\section{Del SIDH a la CVR: EI impacto del caso Martín de Mejía para la investigación de la violencia sexual}

Si bien este caso no fue remitido a la Corte, sino publicado en el Informe Anual de 1996 de la CIDH, el análisis que se realizó fue de suma importancia para la CVR, tanto al momento de la construcción del contexto de violencia sexual en el conflicto peruano, como para resaltar la imposibilidad de denuncia por parte de las víctimas y los altos niveles de impunidad.

Efectivamente, como se ha dicho al inicio, cuando la CVR empezó su trabajo no existían procesos judiciales ni investigaciones concretas sobre los casos de violencia sexual, más allá de algunas denuncias aisladas. La percepción, por tanto, era que no había existido un patrón de violencia sexual contra las mujeres durante el conflicto armado peruano. En ese momento, el Informe Martín de Mejía fue de especial relevancia para el trabajo de la Línea de Género que tuvo a cargo la investigación de la violencia sexual en la CVR.

Como se ha referido, la CIDH utilizó en su análisis tanto informes de mecanismos especiales del Sistema Universal de Protección de Derechos Humanos (como los Relatores Especiales) como otros informes internacionales, los cuales fueron de suma utilidad para el trabajo de la CVR. Asimismo, debido al análisis de los hechos que hizo la CIDH en el marco de la CADH se pudo establecer importantes precedentes tanto en la consideración de la violación sexual como tortura como una afectación a la intimidad de las víctimas, lo cual también fue recogido por la CVR.

La argumentación de la Línea de Género, por tanto, se inició planteando que el informe de la CIDH correspondía al SIDH, del cual el Perú era parte y, por tanto, el seguimiento de sus conclusiones debía ser considerado para la investigación de los hechos por un organismo de justicia transicional como la CVR.

En segundo lugar, el Informe brindaba insumos para la construcción de hipótesis y metodologías de trabajo en la CVR, ya que las fuentes usadas por la CIDH fueron revisadas exhaustivamente $\mathrm{y}$, así, se pudo conseguir información y establecer algunas hipótesis concretas que luego fueron comprobadas durante la investigación de los hechos, referidas, por ejemplo, a las dificultades de denuncia de las víctimas, no solo por el miedo a los agresores, sino por el temor de ser humilladas y estigmatizadas públicamente, a la impunidad generalizada y a la recurrencia de los hechos. Esta información permitió explicar la subrepresentación de los hechos de violencia sexual, la cual había contribuido a consolidar la idea errónea de que estos hechos no se habían producido en la época del conflicto peruano, error que se reafirmaba por la falta de investigaciones específicas y judicialización de casos.

Una segunda hipótesis fue el uso de la violación sexual como una forma de castigo por parte de los agresores, lo cual fue recurrente en los testimonios que llegaron a la CVR y que contribuyó a identificar que no se trataba de actos aislados, sino de un comportamiento generalizado.

En tercer lugar, la obligación de prevenir la violación sexual por parte del Estado también se encuentra en el Informe de la $\mathrm{CIDH}$, ya que se logra probar que había un conocimiento general de que esos hechos se producían y no solo no se tomaban acciones para prevenirlos, sino que tampoco se investigaban adecuadamente. Como se ha dicho, en ese momento no se contaba con los estándares que estableció posteriormente la Corte Interamericana sobre el tema de debida diligencia reforzada que deben tener los Estados en casos de violencia sexual contra las mujeres (Caso López Soto v. Venezuela, 2018), por lo cual el informe de la CIDH es aún de mayor relevancia.

En un contexto difícil donde el trabajo de la CVR no había sido recibido con beneplácito por muchos sectores, explicar la investigación de hechos como la violencia sexual que no se encontraba en el mandato ni sobre el que existían denuncias o condenas, hacía necesario contar con argumentos jurídicos sólidos, y el informe de la CIDH fue de enorme valor para la investigación y, sobre todo, para las víctimas.

En el 2003, la CVR publicó su Informe Final concluyendo, principalmente, que el conflicto armado no había sido vivido de la misma forma por los hombres y las mujeres, que las mujeres no habían podido asumir el rol de protagonistas y víctimas directas, sino que en muchos casos se les consideraba solo como familiares o parejas de las víctimas, y que la violencia sexual (y no solo la violación sexual) no había sido un daño colateral en el conflicto, sino una violación de derechos humanos que afectó mayoritariamente a las mujeres. 
El Informe de la CVR incluyó un capítulo específico sobre "Violencia sexual contra las mujeres" como una forma de reparación a la invisibilización histórica que las mujeres habían sufrido en los estudios e informes sobre el conflicto armado peruano. En este capítulo, la CVR reportó que hubo casos de violencia sexual cometida por todos los actores del conflicto peruano, que ocurrieron en al menos 15 departamentos del Perú y que las víctimas en su gran mayoría eran analfabetas o sólo habían llegado a cursar la primaria, siendo 1984 y 1990 los años de mayor incidencia (Comisión de la Verdad y la Reconciliación, 2004, pág. 374).

Finalmente, la CVR concluyó que

"la violencia sexual contra las mujeres en el contexto de masacres y de ejecuciones arbitrarias, de operativos militares o policiales en medios rurales andinos y amazónicos, aunque también -con menor frecuencia- en medios urbanos, contra personas consideradas sospechosas de tener vínculos con los grupos subversivos, de detenciones arbitrarias fuera del control formal o de hecho del Ministerio Público, de la desaparición forzada de personas, fue una práctica generalizada perpetrada por agentes del Estado. (...) En el caso específico de la violación sexual, la CVR considera que, a la luz de la información recogida, que se trató de una práctica reiterada y persistente que se produjo en el contexto de la violencia sexual antes descrita" (Comisión de la Verdad y la Reconciliación, 2004, pág. 374).

Sin duda, el Informe de la CIDH fue de especial relevancia para las conclusiones de la CVR y, sobre todo, para cambiar la visión que se tenía sobre la ocurrencia de hechos de violencia sexual en ese momento.

\section{Estableciendo el diálogo: Ei impacto de la CVR en la jurisprudencia de la Corte interamericana de Derechos Humanos (La Corte)}

Si se revisan los fallos de La Corte en los años 90, es evidente que esta jurisprudencia no solo no incluía específicamente la violencia sexual, sino que tampoco incorporaba un enfoque de género en la investigación de violaciones de derechos humanos. En este punto, podría argumentarse que la Corte solo puede fallar sobre los casos que le son remitidos por la $\mathrm{CIDH}$ y que casos como el de Raquel Martín de Mejía no le fue enviado en su momento, sino que fue publicado en el Informe de la CIDH.

Sin embargo, lo cierto es que en 1993 la CIDH había remitido a la Corte el caso de la profesora María Elena Loayza Tamayo v. Perú, cuya sentencia se emitió en
$1997^{(16)}$. La señora Loayza Tamayo fue detenida en 1993 por miembros de la División Nacional contra el Terrorismo (en adelante "DINCOTE") acusada ser "presunta colaboradora del grupo subversivo Sendero Luminoso", donde estuvo incomunicada y sin posibilidades de presentar los recursos judiciales correspondientes.

En su demanda ante La Corte, entre otros derechos, la CIDH argumentó que el Estado había violado el derecho a la integridad personal de la peticionaria contenido en el artículo 5 de la CADH, quien había sufrido torturas y malos tratos durante la detención, incluyendo violación sexual. Sin embargo, en su sentencia de 1997, la Corte estableció que

"Aun cuando la Comisión alegó en su demanda que la víctima fue violada durante su detención, la Corte, después de analizar el expediente $y$, dada la naturaleza del hecho, no está en condiciones de darlo por probado. Sin embargo, los otros hechos alegados como la incomunicación durante la detención, la exhibición pública con un traje infamante a través de medios de comunicación, el aislamiento en celda reducida, sin ventilación ni luz natural, los golpes y otros maltratos como el ahogamiento, la intimidación por amenazas de otros actos violentos, las restricciones al régimen de visitas, constituyen formas de tratos crueles, inhumanos o degradantes en el sentido del artículo 5.2. de la Convención Americana" [énfasis agregado]

Cabe preguntarse cuáles fueron los criterios de la Corte para admitir la existencia de los malos tratos y torturas sufridos por la víctima y no la violación sexual. La Corte no precisa a qué se refiere con "la naturaleza del hecho" y considera probados todos los demás hechos, pero no la agresión sexual. Como se ha dicho, en ese momento la investigación de violencia sexual como una violación de derechos humanos y el enfoque de género en los estándares jurisprudenciales internacionales no tenían el desarrollo que hoy tienen. Si bien La Corte condena al Estado por violaciones al derecho al debido proceso y a la integridad personal, lo cierto es que la violación sexual resultó invisibilizada.

(16) Corte Interamericana de Derechos Humanos, caso María Elena Loayza v. Peru, 1997, http://www.corteidh.or.cr/docs/casos/articulos/ seriec_33_esp.pdf 
En la década del 2000 las cosas van cambiando. En el 2004, La Corte dio la sentencia del Caso Masacre Plan de Sánchez vs. Guatemala, en donde hizo referencia al Informe de la Comisión para el Esclarecimiento Histórico de Guatemala que se señala que "la violación sexual de las mujeres fue una práctica común dirigida a destruir la dignidad de la persona en uno de sus aspectos más íntimos y vulnerables"(17), estableciéndose que "las comunidades mismas quedaron afectadas por esta práctica, que se convirtió en motivo de vergüenza colectiva"(18). Es importante la forma como la Corte analiza la situación de las mujeres en este caso, las cuales fueron "estigmatizadas en sus comunidades y han sufrido por la presencia de los victimarios en las áreas comunes del municipio"(19).

Sin embargo, será en el 2006, con la sentencia del Caso del Penal Miguel Castro vs. Perú(20), referido al uso excesivo de la fuerza del Estado en el marco de un operativo en dicho penal, donde la Corte marque el inicio de una tendencia jurisprudencial que visibiliza la violencia sexual y el análisis de género de las violaciones de derechos humanos, verificándose el diálogo entre avances nacionales y estándares interamericanos.

En primer lugar, a lo largo de su sentencia, la Corte hizo referencia explícita a la CVR y a su Informe Final y, para el caso concreto de la violencia sexual, la Corte sostuvo:

"También es preciso resaltar que en el referido informe final de la CVR se analizó que, dentro de ese contexto de violaciones a los derechos humanos durante el conflicto interno, las mujeres se vieron afectadas por la violencia de manera diferente a los hombres. La CVR incluyó en su informe un capítulo específico sobre la violencia sexual contra las mujeres y también se refirió a la situación que experimentaron las madres recluidas en centros penitenciarios. Asimismo, en dicho informe se concluyó que durante el conflicto interno y con motivo de este los agentes estatales fueron responsables de aproximadamente un $83 \%$ de los casos de violación sexual contra las mujeres" (Corte IDH, 2006, párr. 206).

Este es un primer aporte de suma importancia, ya que, debido al Informe de la CVR, la Corte da por probado el contexto de violencia sexual existente en el Perú en el marco del conflicto armado interno. Asimismo, la Corte recoge el análisis diferenciado por género que la CVR había procurado en su trabajo y su Informe Final, lo cual refuerza la importancia de este tipo de enfoque en las investigaciones de derechos humanos. Como señala la sentencia:

“(...) en su Informe Final la Comisión de la Verdad y Reconciliación del Perú afirmó que en el conflicto armado existió "una práctica (...) de violaciones sexuales y violencia sexual contra mujeres principalmente", la cual "es imputable (...) en primer término a agentes estatales (...) y en menor medida a miembros de los grupos subversivos". Asimismo, la CVR señaló que durante el referido conflicto los actos de violencia sexual contra las mujeres tenían como objetivos castigar, intimidar, presionar, humillar y degradar a la población" (Corte IDH, 2006, párr. 225).

Al darse por probado el contexto de violencia sexual, los casos de violencia sexual ocurridos en el marco temporal y en las circunstancias que identificó la CVR, deben ser considerados como violaciones de derechos humanos. Recordemos, por ejemplo, el caso María Elena Loayza Tamayo vs. Perú anteriormente referido y reflexionemos sobre el impacto que hubiera tenido en la sentencia si el contexto de violencia sexual en el conflicto peruano hubiera sido probado en ese momento.

El análisis de género abunda en la sentencia Castro Castro, por ejemplo, al momento en que la Corte analiza el impacto de la violencia sufrida por internos e internas, señalando que:

“(..) las mujeres se vieron afectadas por los actos de violencia de manera diferente a los hombres, que algunos actos de violencia se encontraron dirigidos específicamente a ellas y otros les afectaron en mayor proporción que a los hombres. Ha sido reconocido por diversos órganos peruanos e internacionales que durante los conflictos armados las mujeres enfrentan situaciones específicas de afectación a sus derechos humanos, como lo son los actos de violencia sexual, la cual en muchas ocasiones es utilizada como un medio simbólico para humillar a la parte contraria" (Corte IDH, 2006, párr. 223 y 224).

Adicionalmente, La Corte IDH (2006) hace un análisis de género de las condiciones de detención, señalando que

"la incomunicación severa tuvo efectos particulares en las internas madres. Diversos órganos internacionales han enfatizado la obligación de los Estados de tomar en

(17) Ver Corte IDH, Caso Masacre Plan de Sánchez vs. Guatemala, (Reparaciones), Sentencia de 19 de noviembre de 2004, Serie C No. 116, párr. 38. Recuperado de: http://www.corteidh.or.cr/docs/casos/articulos/seriec_116_esp.pdf

(18) Ibidem, párr. 38

(19) Ibidem, párr. 49.19.

(20) Ver Corte IDH, Caso del Penal Miguel Castro Castro vs. Perú, (Fondo, Reparaciones y Costas), Sentencia de 25 de noviembre de 2006, Serie C N ${ }^{\circ} 160$. Recuperado de: http://www.corteidh.or.cr/docs/casos/articulos/seriec_160_esp.pdf 
consideración la atención especial que deben recibir las mujeres por razones de maternidad, lo cual implica, entre otras medidas, asegurar que se lleven a cabo visitas apropiadas entre madre e hijo" (párr. 330).

Asimismo, la Corte se refiere al caso de las mujeres embarazadas, resaltando que "además de haber visto lesionada su propia integridad física, padecieron sentimientos de angustia, desesperación y miedo por el peligro que corría la vida de sus hijos" (Corte IDH, 2006, párr. 292).

Un tema fundamental es que la Corte analiza las diferentes formas de violencia sexual, sin limitarse al enfoque tradicional que solo prioriza la violación sexual. Así, la Corte se refiere a los desnudos forzados que sufrieron las internas a las que además se les obligó a utilizar los baños acompañadas de un guardia armado quien permanentemente las amenazaba con un arma. Ante ello, la Corte señala que "la violencia sexual se configura con acciones de naturaleza sexual que se cometen en una persona sin su consentimiento, que además de comprender la invasión física del cuerpo humano, pueden incluir actos que no involucren penetración o incluso contacto físico alguno". (Corte IDH, 2006, párr. 306).

En el mismo sentido, resulta de vital importancia que la Corte haya identificado como violación sexual que configura tortura la inspección vaginal sufrida por una interna en el Hospital de la Sanidad de la Policía por parte de un grupo de personas encapuchadas (Caso Castro Castro v. Perú, 2006, párr. 309), dejando de lado visiones tradicionales que limitaban la violación a las relaciones sexuales sin consentimiento y estableciendo que por "violación sexual también debe entenderse actos de penetración vaginales o anales, sin consentimiento de la víctima, mediante la utilización de otras partes del cuerpo del agresor u objetos, así como la penetración bucal mediante el miembro viril" (Corte IDH, 2006, párr. 310).

Asimismo, la Corte estableció que la violencia sexual "se configura con acciones de naturaleza sexual que se cometen contra una persona sin su consentimiento, que además de comprender la invasión física del cuerpo humano, pueden incluir actos que no involucren penetración o incluso contacto físico alguno" (Corte IDH, 2006, párr.306), estableciendo una definición amplia sobre el tema.

A partir de este caso, la Corte fue consolidando una tendencia jurisprudencial orientada al reconocimiento de la violencia sexual como una situación de violación de derechos humanos y consolidando el enfoque de género en sus posteriores sentencias. Así, en el caso González y Otras ("Campo Algodonero") vs. México (2009), la Corte concluyó que los homicidios de mujeres presentaban altos grados de violencia, incluyendo la sexual, y habían sido influenciados por una cultura de discriminación contra la mujer, la cual había incidido en los motivos, en la modalidad de los crímenes y en la respuesta de las autoridades frente a estos ${ }^{(21)}$.

En el 2010, en los casos Rosendo Cantú y Otra y Fernández Ortega y Otros, ambos contra México (2010), La Corte enfatizó que "la violación sexual es una experiencia sumamente traumática que tiene severas consecuencias y causa gran daño físico y psicológico que deja a la víctima humillada física y emocionalmente"(22). Por tanto, la Corte concluyó que el sufrimiento severo de la víctima es inherente a la violación sexual, pese a no existir evidencia de lesiones o enfermedades físicas ya que las víctimas experimentan daños y secuelas psicológicas y sociales ${ }^{(23)}$.

Posteriormente, en el caso Espinoza Gonzáles vs. Perú (2014), la Corte se refirió al caso de las mujeres detenidas, reiterando lo dicho en el caso Castro Castro, en el sentido de que "no deben sufrir discriminación, y deben ser protegidas de todas las formas de violencia o explotación". Dicha discriminación incluye "la violencia dirigida contra la mujer porque es mujer o que la afecta en forma desproporcionada", y abarca "actos que infligen daños o sufrimientos de índole física, mental o sexual, amenazas de cometer esos actos, coacción y otras formas de privación de la libertad" (Corte IDH, 2014, párr. 223).

Recientemente, la Corte dio dos nuevos precedentes en esta línea jurisprudencial sobre violencia sexual. Por un lado, en el caso Atenco vs. México (2018) $)^{(24)}$, la Corte sostuvo que la ausencia de una perspectiva de género en la investigación de la tortura

(21) Ver Corte IDH, Caso Gonzáles y Otras (“Campo Algodonero”) vs. México, (Excepción Preliminar, Fondo, Reparaciones y Costas), Sentencia de 16 de noviembre de 2009, Serie C No. 205, párr. 164. Recuperado de: http://www.corteidh.or.cr/docs/casos/articulos/ seriec_205 esp.pdf

(22) Ver Corte IDH, Caso Fernández Ortega vs. México, (Excepción Preliminar, Fondo, Reparaciones y Costas), Sentencia de 30 de agosto de 2010, Serie C No. 215, párr. 124.

(23) Ibidem.

(24) Caso Mujeres víctimas de tortura sexual en Atenco vs. México, Sentencia de 28 de noviembre de 2018 (Excepción Preliminar, Fondo, Reparaciones y Costas) http://www.corteidh.or.cr/docs/casos/articulos/seriec_371_esp.pdf 
y violencia sexual violaba las obligaciones especiales impuestas por la Convención de Belém do Pará y el deber de respetar y garantizar sin discriminación los derechos contenidos en la Convención Americana de Derechos Humanos.

Por otro lado, en el Caso López Soto y otros vs. Venezuela (Corte IDH, 2018) sobre tortura, en el que nuevamente hace referencia a la Convención de Belém do Pará, afirmando que este texto "debe permear la interpretación evolutiva de las conductas y actos de violencia contra la mujer que pueden encuadrarse como tortura" (25). Por ello, la Corte señala "que actos intencionales que acarrean a la mujer sufrimientos graves de carácter físico, sexual o psicológico cometidos por un particular pueden configurar actos de tortura y merecen un reproche adecuado a su gravedad para alcanzar el objetivo de su erradicación"(26). Este aporte, como se puede ver, es fundamental ya que hace referencia explícita a los actos de particulares que pueden, eventualmente, configurar tortura.

Sin duda, en los próximos años los estándares jurídicos interamericanos en materia de violencia sexual seguirán ampliándose y garantizando una mayor protección a las víctimas.

\section{Conclusiones}

Como se ha visto, el diálogo entre el SIDH y los avances nacionales en materia de enfoque de género e investigación sobre la violencia sexual contra las mujeres, ha sido parte del proceso de consolidación de un estándar jurídico adecuado que, progresivamente, ha ido reconociendo la violencia sexual como una violación de derechos humanos $y$ ha incorporado el enfoque de género en el estudio e investigación de las violaciones de derechos humanos.

En esta línea, hace poco la CIDH emitió por primera vez en su historia una medida cautelar ${ }^{(27)}$ referida a un caso colombiano de tentativa de feminicidio y en el cual, luego de revisar los hechos, la CIDH concluyó que la víctima y su familia se encontraban "en una situación de gravedad y urgencia, toda vez que sus derechos enfrentan un riesgo de daño irreparable", por lo que solicitó al Estado de Colombia "que adopte las medidas necesarias para proteger sus derechos a la vida e integridad, con perspectiva de género olos enfoques diferenciados que resulten pertinentes, de conformidad con los estándares y obligaciones internacionales aplicables" [énfasis agregado] ${ }^{(28)}$. Hace unos años, hubiera resultado imposible pensar que una medida de este tipo se hubiera dado y, sin embargo, hoy comprobamos que un enfoque de género y un diálogo con las realidades locales permite la construcción de estándares de mayor protección hacia las víctimas.

\section{Referencias bibliográficas}

Amnesty International (1 de noviembre de 1991a). Perú: Human Rights in a Climate of Terror. Amnesty International. Recuperado de https:// www.amnesty.org/en/documents/amr46/056/1991/en/

(1991b). Women in the Front Line: Human Rights Violations against Women. Nueva York: Amnesty International Publications.

Brooke, James (29 de abril de 1993). Rapists in Uniform: Peru Looks the Other Way. The New York Times. Recuperado de https://www. nytimes.com/1993/04/29/world/lima-journal-rapists-in-uniform-perulooks-the-other-way.html

Caretas (11 de marzo de 1993). Violencia Sexual.

Caso Castro Castro vs. Perú (Corte Interamericana de Derechos Humanos, 25 de noviembre de 2006). Recuperado de http://www. corteidh.or.cr/docs/casos/articulos/seriec_160_esp.pdf

Caso Espinoza Gonzáles vs. Perú (Corte Interamericana de Derechos Humanos, 20 de noviembre de 2014). Recuperado de http://www. corteidh.or.cr/docs/casos/articulos/seriec_289_esp.pdf

Caso López Soto y otros vs. Venezuela (Corte Interamericana de Derechos Humanos, 26 de septiembre de 2018). Recuperado de http:// www.corteidh.or.cr/docs/casos/articulos/seriec_362_esp.pdf

Caso María Elena Loayza vs. Perú (Corte Interamericana de Derechos Humanos, 17 de septiembre de 1997) Recuperado de http://www. corteidh.or.cr/docs/casos/articulos/seriec_33_esp.pdf

Comisión de la Verdad y Reconciliación (CVR) (2004). Informe final de la Comisión de la Verdad y Reconciliación del Perú (Tomo VI). Lima: CVR. Recuperado de http://cverdad.org.pe/ifinal/

Comisión Interamericana de Derechos Humanos (1996). Caso Raquel Martin de Mejía v. Perú, Caso 10.970 Informe No. 5/96, InterAm.C.H.R., OEA/Ser.L/V/II.91 Doc. 7 at 168 (1996). Recuperado de http://hrlibrary.umn.edu/cases/1996/Speru5-96.htm

Human Rights Watch, Americas Watch and the Women's Rights Project (1992). Untold Terror: Violence Against Women in Peru's Armed Conflict. New York: Human Rigths Watch.

(25) Corte IDH, Caso Linda López Soto y Otros vs. Venezuela, (Fondo, Reparaciones y Costas), Sentencia de 26 de septiembre de 2018, Serie C No. 362, párr. 197, http://www.corteidh.or.cr/docs/casos/articulos/seriec_362_esp.pdf

(26) Ibidem, párr. 194.

(27) Comisión Interamericana de Derechos Humanos, Resolución 9/20, MC 1212-19 - M.I.F.M. y familia, Colombia, 5 de febrero del 2020. 